\title{
A Note on the Influence of Intermediate Restraints and Hinges in Frequencies and Mode Shapes of Beams
}

\author{
Javier L. Raffo \\ Universidad Tecnológica Nacional, Facultad Regional Delta, San Martín 1171, 2804 Campana, Argentina \\ Ricardo O. Grossi \\ Universidad Nacional de Salta, INIQUI - Facultad de Ingeniería, Av. Bolivia 5150, 4400 Salta, Argentina
}

(Received 21 December 2012; revised: 27 March 2013; accepted: 30 April 2013)

\begin{abstract}
This note deals with the free transverse vibration of a beam with two arbitrarily located internal hinges, four intermediate elastic restraints, and ends elastically restrained against rotation and translation. The method of separation of variables is used for the determination of the exact frequencies and mode shapes. New results are presented for different boundary conditions and restraint conditions in the internal hinges.

The mathematical model is also used to study the influence on the frequencies and mode shapes of varying intermediate supports that are located at the nodal points of higher modes. A detailed numerical study on the effects of the locations of intermediate translational restraints and their stiffness on the natural frequencies and mode shapes is performed for different boundary conditions. The effect of the presence of the internal hinges is also analysed. Graphs and tables of the non-dimensional frequencies and the corresponding mode shapes are given in order to illustrate the behaviour of frequency parameters and the presence of mode shape switching.
\end{abstract}

\section{INTRODUCTION}

There has been extensive research into the vibration of Euler-Bernoulli beams with elastic restraints. It is not possible to give a reasonable and detailed account of this great amount of information; nevertheless, some relevant references will be cited. Particularly, several investigators have studied the influence of elastic restraints at the ends of vibrating beams. ${ }^{1-16}$ Exact frequency and normal mode shape expressions have been derived for uniform beams with elastically restrained ends against rotation and translation. ${ }^{17}$ Excellent handbooks have appeared in the literature giving frequencies, tables and mode shape expressions. ${ }^{18,19}$

The problem of the vibrations of beams that are elastically restrained at intermediate points has also been extensively treated. One of the earliest works was performed by Lee and Saibel who analysed the problem of free vibrations of a constrained beam with intermediate elastic supports. ${ }^{20}$ Rutemberg presented eigenfrequencies for a uniform cantilever beam with a rotational restraint at an intermediate position. ${ }^{21}$ Lau extended Rutemberg's results with an additional translational restriction. ${ }^{22}$ Maurizi and Bambill analysed the transverse vibrations of clamped beams with an intermediate translational restraint. ${ }^{23}$ Rao analysed the frequencies of a clamped-clamped uniform beam with intermediate elastic support. ${ }^{24}$ De Rosa et al. studied the free vibrations of stepped beams with intermediate elastic supports. ${ }^{25}$ Ewing and Mirsafian analysed the forced vibrations of two beams joined with a non-linear rotational joint. ${ }^{26}$ Arenas and Grossi presented exact and approximate frequencies of a uniform beam, with one end spring- hinged and a rotational restraint in a variable position. ${ }^{27}$ Grossi and Albarracín determined the exact eigenfrequencies of a uniform beam with intermediate elastic constraints. ${ }^{28}$

The minimum stiffness of an elastic traslational restriction that raises a natural frequency of a beam to its upper limit has been investigated by several researchers. Courant and Hilbert have demonstrated that the optimum location of a rigid support should be at the nodal points of a higher vibration mode. ${ }^{29}$ Akesson and Olhoff showed that in the case of elastic supports the optimum locations are the same as that of rigid supports, and there exists a minimum stiffness of an additional elastic support whenever the fundamental frequency of a uniform cantilever beam is increased to its maximum. ${ }^{30}$ Wang determined the minimum stiffness of an internal elastic support to maximize the fundamental frequency of a vibrating beam. ${ }^{31}$ Wang et al. derived the closed-form solution for the minimum stiffness of a simple point support that raises a natural frequency of a beam to its upper limit. ${ }^{32}$ Albarracín et al. detected a rather curious situation of changes in frequency values and mode shapes when an intermediate translational restraint is placed in a beam that is simply supported at both ends. ${ }^{33}$

There is only a limited amount of information for the vibration of beams with internal hinges. Wang and Wang studied the fundamental frequency of a beam with an internal hinge with an axial force. ${ }^{34}$ Chang et al. investigated the dynamic response of a beam with an internal hinge, subjected to a random moving oscillator. ${ }^{35}$ Grossi and Quintana investigated the natural frequencies and mode shapes of a non homogeneous tapered beam subjected to general axial forces, with an arbitrarily located internal hinge and elastic supports and ends that 